University of Tennessee Health Science Center UTHSC Digital Commons

\title{
Interaction Between Two E3 ligases, NEDD8ylated Cullin and HHARI
}

Kheewoong Baek

University of Tennessee Health Science Center

Follow this and additional works at: https://dc.uthsc.edu/dissertations

Part of the Enzymes and Coenzymes Commons, Medical Biochemistry Commons, and the Medical Immunology Commons

\section{Recommended Citation}

Baek, Kheewoong (http://orcid.org/0000-0002-6853-4936), "Interaction Between Two E3 ligases, NEDD8ylated Cullin and HHARI" (2016). Theses and Dissertations (ETD). Paper 392. http://dx.doi.org/ 10.21007/etd.cghs.2016.0401.

This Thesis is brought to you for free and open access by the College of Graduate Health Sciences at UTHSC Digital Commons. It has been accepted for inclusion in Theses and Dissertations (ETD) by an authorized administrator of UTHSC Digital Commons. For more information, please contact jwelch30@uthsc.edu. 


\title{
Interaction Between Two E3 ligases, NEDD8ylated Cullin and HHARI
}

\begin{abstract}
RBR (RING1-in between RING-RING2) is a special type of E3 ubiquitin ligase containing three zinc-binding RING (Really Interesting New Gene) domains, while adopting mechanisms of HECT (Homologous to E6-AP Carboxyl Terminus) for substrate ubiquitination. Most well known RBRs include Parkin and HOIP, which are associated with Parkinson's disease and innate immune deficiency. However, it is not well known how the RBR proteins gain activity, as they are known to be autoinhibited. Here I show that a specific F430A, E431A, E503A triple mutation of RBR protein HHARI (Human homologue of Ariadne) and its interaction with NEDD8ylated cullin RING ligase can both boost its activity and stabilize complex formation. Analytical size-exclusion chromatography, autoubiquitination, and electron microscopy reveal consistent behavior for this triple-mutant. Future structure-based studies will help elucidate the mechanism of the unsolved mystery of RBR activation and its interaction with NEDD8ylated cullin RING ligases.
\end{abstract}

\section{Document Type}

Thesis

Degree Name

Master of Science (MS)

Program

Biomedical Sciences

Research Advisor

Brenda A. Schulman, Ph.D.

Keywords

ubiquitin

\section{Subject Categories}

Chemicals and Drugs | Enzymes and Coenzymes | Medical Biochemistry | Medical Immunology | Medical Sciences | Medicine and Health Sciences 
Interaction Between Two E3 Ligases, NEDD8ylated Cullin and HHARI

\author{
A Dissertation \\ Presented for \\ The Graduate Studies Council \\ The University of Tennessee \\ Health Science Center
}

\author{
In Partial Fulfillment \\ Of the Requirements for the Degree \\ Master of Science \\ From The University of Tennessee
}

By

Kheewoong Baek

May 2016 
Copyright (C) 2016 by Kheewoong Baek.

All rights reserved. 


\section{ACKNOWLEDGEMENTS}

I would like to thank Dr. Brenda Schulman for providing me the opportunity to experience cutting edge science in structural biology.

I would also like to thank my committee members Dr. Eric Enemark and Dr. Julio Cordero-Morales for providing great feedback, support, and for their time.

I would like to thank all the lab members of the Schulman Lab, they are fantastic people fun to work with, and I learn a lot from everyone everyday.

I would also like to thank my parents back home giving me support in every way. 


\begin{abstract}
RBR (RING1-in between RING-RING2) is a special type of E3 ubiquitin ligase containing three zinc-binding RING (Really Interesting New Gene) domains, while adopting mechanisms of HECT (Homologous to E6-AP Carboxyl Terminus) for substrate ubiquitination. Most well known RBRs include Parkin and HOIP, which are associated with Parkinson's disease and innate immune deficiency. However, it is not well known how the RBR proteins gain activity, as they are known to be autoinhibited. Here I show that a specific F430A, E431A, E503A triple mutation of RBR protein HHARI (Human homologue of Ariadne) and its interaction with NEDD8ylated cullin RING ligase can both boost its activity and stabilize complex formation. Analytical size-exclusion chromatography, autoubiquitination, and electron microscopy reveal consistent behavior for this triple-mutant. Future structure-based studies will help elucidate the mechanism of the unsolved mystery of RBR activation and its interaction with NEDD8ylated cullin RING ligases.
\end{abstract}




\section{TABLE OF CONTENTS}

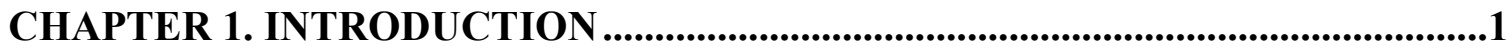

The Ubiquitin Pathway .................................................................................... 1

Cullin RING Ligases .................................................................................... 1

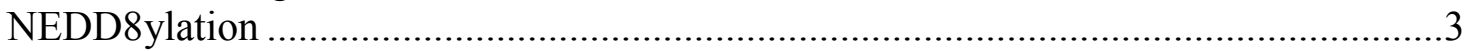

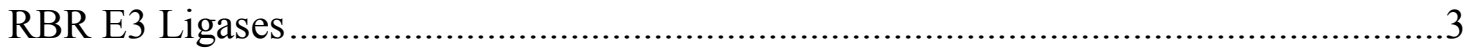

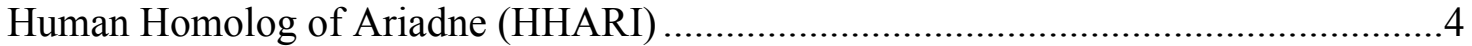

Interaction Between HHARI and Cullin RING Ligase .......................................4

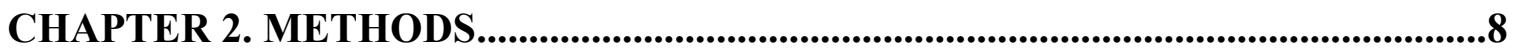

Protein Expression and Purification ...................................................................

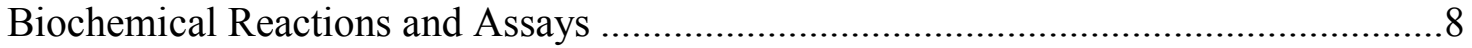

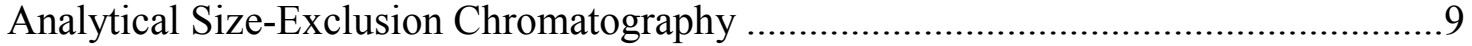

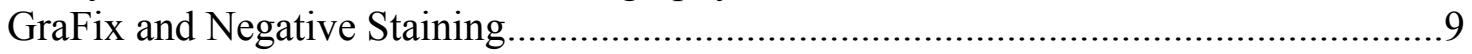

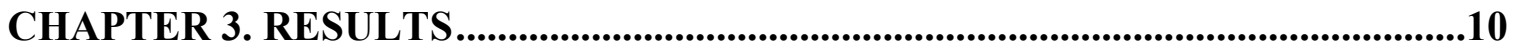

NEDD8ylated Cullin RING Ligases Activate HHARI ............................................... 10

NEDD8ylated Cullin RING Ligases Bind with HHARI ........................................... 10

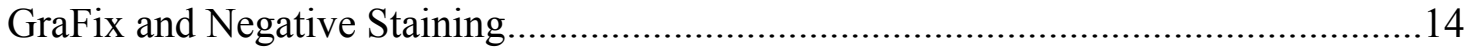

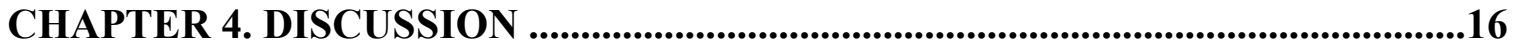

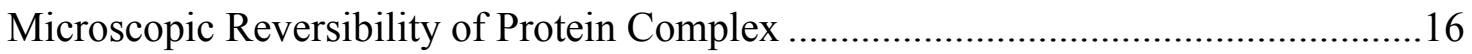

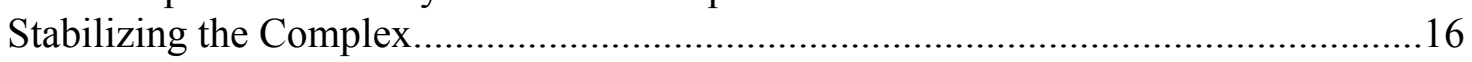

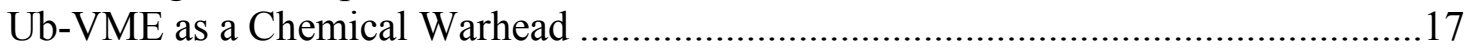

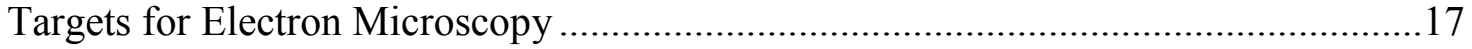

LIST OF REFERENCES ......................................................................................................19

VITA 


\section{LIST OF FIGURES}

Figure 1-1. The Ubiquitin Pathway. .........................................................................2

Figure 1-2. Human Homologue of Ariadne.....................................................................5

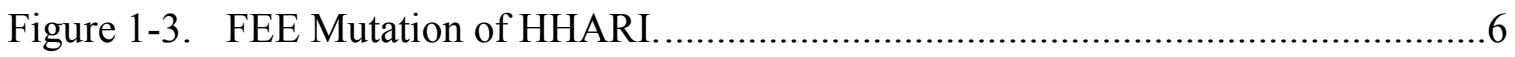

Figure 3-1. FEE Mutation of HHARI and the Addition of N8Cul1Rbx1 Both Activate HHARI.............................................................................. 11

Figure 3-2. Analytical Size-Exclusion Chromatography Shows Interaction Between

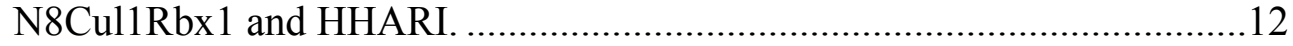

Figure 3-3. Interaction Between N8Cul3Rbx1 and HHARI. ....................................13

Figure 3-4. Electron Microscopy of HHARI FEE, N8Cul1Rbx1, and Skp1/Fbw7.......15 


\section{LIST OF ABBREVIATIONS}

$\begin{array}{ll}\text { CRL } & \text { Cullin RING ligase } \\ \text { DTT } & \text { 1,4-Dithiothreitol } \\ \text { HECT } & \text { Homologous to E6-AP Carboxyl Terminus } \\ \text { HHARI } & \text { Human homologue of Ariadne } \\ \text { NEDD8 } & \text { Neural precursor cell expressed developmentally down-regulated } 8 \\ \text { N8Cul1Rbx 1 } & \text { NEDD8ylated Cullin-1 Rbx1 } \\ \text { N8Cul3Rbx1 } & \text { NEDD8ylated Cullin-3 Rbx1 } \\ \text { RBR } & \text { RING1- in between RING- RING2 } \\ \text { Rbx } & \text { RING box protein } \\ \text { RING } & \text { Really Interesting New Gene } \\ \text { WT } & \text { Wild type } \\ \text { Ub } & \text { Ubiquitin } \\ \text { Ub-VME } & \text { Ubiquitin Vinyl Methyl Ester }\end{array}$




\section{CHAPTER 1. INTRODUCTION}

\section{The Ubiquitin Pathway}

Ubiquitination is a post-translational covalent modification of protein by a 76 amino acid ubiquitin ( $\mathrm{Ub}$ ) forming an isopeptide bond between the ubiquitin $\mathrm{C}$-terminal glycine residue 76 and the substrate lysine. Ubiquitin can form monoubiquitin or polyubiquitin chains linked by the N-terminal methionine or seven lysine residues (K6, $\mathrm{K} 11, \mathrm{~K} 27, \mathrm{~K} 29, \mathrm{~K} 33, \mathrm{~K} 48, \mathrm{~K} 63$ ) and its C-terminus GG is a required for its covalent modification to proteins (Hodgins et al., 1992; Komander, 2009). Different types of polyubiquitin chains allow participation of substrates in many important processes including proteosomal/lysosomal degradation, transcription, signal transduction, and protein trafficking (Komander and Rape, 2012).

In order to ubiquitinate target substrates, the pathway involves three sequential mechanisms (Figure 1-1). First, the C-terminus of ubiquitin must be activated by acyladenylation in the presence of ATP and $\mathrm{Mg}^{2+}$. The catalytic cysteine of the E1 activating enzyme attacks the adenylated ubiquitin, producing a highly active E1 Ub thioester complex (Schulman and Harper, 2009). Then, the E2 ubiquitin conjugating enzyme accepts the ubiquitin from the E1 by transthioesterification. The ubiquitin-bound E2 interacts with the E3 ubiquitin ligase, which ultimately ubiquitinates target protein substrates (Streich and Lima, 2014). This step largely divides into two major categories of E3s. First, RING (Really New Interesting Gene) E3 ligases act as scaffolds for the E2 Ub and substrates, where the ubiquitin directly transfers to the lysine of substrate protein. Over 600 E3 ligases are known to be part of the RING family (Deshaies and Joazeiro, 2009). Second, HECT (Homologous to E6-AP Carboxyl Terminus) E3 ligases also act as scaffolds for the E2 Ub and substrates, but makes a thioester linked E3 Ub intermediate, where the Ub from the E2 transfers to the catalytic cysteine of the HECT E3, and then sequentially transfers the Ub to the substrate protein via its HECT domain (Huibregtse et al., 1995). There is a hybrid type of E3 ligases called RBRs (RING1-in between RING-RING2) where it contains RING domains but utilizes HECT mechanisms. Overall in the human genome, there are two E1s, tens of E2s, and hundreds of E3s that regulate ubiquitination of over thousands of substrates.

\section{Cullin RING Ligases}

Cullin RING ligases (CRL), a superfamily of RING E3s, are modular complexes composed of a catalytic RING subunit bound to cullin repeats. The RING domain interacts with two zinc ions by its cysteine and histidine residues to provide structural stability for protein-protein interactions by producing a globular platform (Borden et al., 1995). Each different cullins have variety of substrate receptors that allow participation of cullin RING ligases in majority of cellular processes (Deshaies and Joazeiro, 2009).

$\mathrm{Cul1}, \mathrm{Cul} 2, \mathrm{Cul} 3, \mathrm{Cul} 4 \mathrm{~A}, \mathrm{Cul} 4 \mathrm{~B}$, and Cul5 along with their substrate receptors and RING box proteins $(\mathrm{Rbx})$ yield around 500 different combinations of cullin RING ligases 


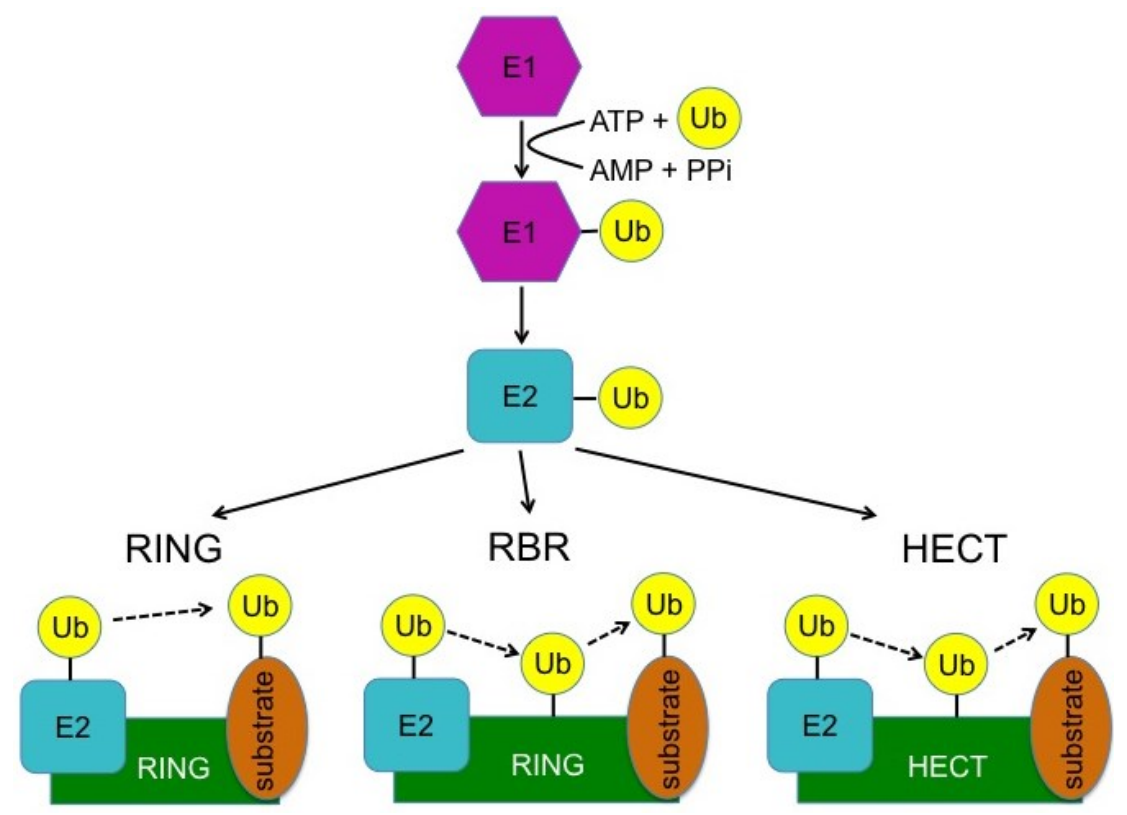

Figure 1-1. The Ubiquitin Pathway.

Schematic of the ubiquitin pathway. Catalytic cysteine of E1 attacks adenylated ubiquitin, producing a highly reactive complex of E1 Ub. By transthioesterification, ubiquitin transfers from E1 to E2, and further onto substrate via 3 types of E3 ubiquitin ligases: RING, HECT, and RBR. 
(Zimmerman et al., 2010). Among the CRL family includes the well-known Skp1-Cul1F-box (SCF) ubiquitin ligase where the N-terminus of Cull binds Skp1, and the Cterminus binds Rbx 1 for E2 interaction. The F-box protein directly binds to Skp1 by the F-box domain for substrate specificity (Cardozo and Pagano, 2004). The F-box proteins also contain protein-protein interaction motifs such as leucine-rich repeats (LRR) or WD repeats (Schulman et al., 2000). By different types of F-box proteins, the cullin RING ligases undergo different conformations. For instance, Fbw7 can dimerize Cul1Rbx1 complex by its D-domain, which is highly conserved in F-box proteins with WD40 repeat motifs (Tang et al., 2007). Cul3Rbx 1 can also dimerize via MATH-BTB proteins (Zhuang et al., 2009).

\section{NEDD8ylation}

Many ubiquitin like proteins (Ubl) exist to regulate protein functions. NEDD8 (neural-precursor-cell-expressed developmentally down-regulated 8), a Ubl, is essential for the activity of cullin RING ligases. Human NEDD8 shares $60 \%$ sequence identity with ubiquitin and has a NEDD8ylation cascade. By its Nedd8 E1 APPBP1-Uba3, Nedd8 E2 Ubc12, and Nedd8 E3 Rbx1/2, Nedd8 is ultimately attached onto its substrate cullin (Huang et al., 2009). With its E3 RING box protein, NEDD8 conjugates onto the cullin scaffold lysine that activates the cullin RING ligase (Lydeard et al., 2013). Cullin NEDD8ylation enhances E2 recruitment by its selectivity towards ubiquitin charged E2s and improves substrate ubiquitination (Saha and Deshaies, 2008). NEDD8 ligation also causes a dramatic conformational change of the Cullin Rbx complex so that the E2 Ub positions proximally to the substrate lysine (Duda et al., 2008; Scott et al., 2014). DCN1 also enhances cullin NEDD8ylation as an auxiliary E3 by binding its PONY (potentiating NEDD8) domain with Cul1 and Ubc12. NEDD8ylated cullins are deNEDD8ylated by the multiprotein complex COP9 signalosome (CSN) (Enchev et al., 2012; Lyapina et al., 2001). Recent structural studies were able to observe the mechanism of CSN and its cullin RING ligase regulation (Cavadini et al., 2016; Mosadeghi et al., 2016). DeNEDD8ylation by CSN blocks substrate access and allows substrate receptor exchange by CAND1. CAND1 can only bind to the unNEDD8ylated form of cullins and this substrate receptor exchange allows cullin RING ligases to be involved in numerous substrate ubiquitinations (Pierce et al., 2013; Schmidt et al., 2009).

\section{RBR E3 Ligases}

There is a special family of E3 ligases, called RBRs (RING1-in between RINGRING2). RBRs are reported to be RING/HECT hybrid E3s. Unlike RING E3s, RBRs produce an E3 Ub intermediate, where the ubiquitin makes a thioester link on the catalytic cysteine of RING2, just as would occur on a HECT E3 (Wenzel et al., 2011). RBR E3s contain 3 RING domains each coordinating two zinc ions: RING1, in-betweenRING, and RING2 (Hristova et al., 2009). However, the name today is controversial as the RING2 does not have the canonical RING E3 structure with only a single cysteine. So far, only 13 human RBRs and 2 yeast RBRs have been identified (Eisenhaber et al., 
2007). Most studied RBRs include the famous Parkinson's disease related Parkin, LUBAC (linear ubiquitin chain assembly complex), HOIP, HOIL-1L, Cul9, HHARI, and Triad1 (Spratt et al., 2014). Studies show that RBRs adopt an autoinhibitory conformation, where a specific domain masks the catalytic cysteine of RING2: Ub1 domain for Parkin and Ariadne domain for HHARI (Chaugule et al., 2011; Duda et al., 2013; Trempe et al., 2013; Wauer and Komander, 2013). RBRs are also known to interact with kinases. PINK1(PTEN induced putative kinase 1) is reported to phosphorylate ubiquitin, sequentially activating parkin (Koyano et al., 2014).

\section{Human Homolog of Ariadne (HHARI)}

Human homolog of Ariadne, HHARI, is an E3 RBR ligase consisting of an Nterminal acidic/glycine rich domain, UBA domain, RING1, IBR, RING2, and an Ariadne domain (Figure 1-2). In recent years, Duda et al were able to solve the crystal structure of HHARI, which provides tremendous insight into the mechanism of RBRs. The structure explains how HHARI is autoinhibited, as 30\% of the RING2 surface including the catalytic cysteine is masked by the Ariadne domain (Figure 1-3). In order to study the activation of HHARI, Duda et al performed various combinations of mutational analyses, and discovered a set of mutations that significantly activates HHARI. Triple mutation of F430A, E431A, and E503A significantly activated HHARI by a autoubiquitination assay, which is known to be a good measurement of E3 ligase activity (Duda et al., 2013). Seen in the structure (PDB ID: 4KBL), all three residues closely interact with the catalytic cysteine residue 357 , indicating that mutating these residues might facilitate opening of the RING2 for ubiquitin access.

\section{Interaction Between HHARI and Cullin RING Ligase}

Kelsall et al screened combinations of two E3 ligases, one RBR and one RING, for activity. With several combinations, they found that NEDD8ylated cullin RING ligases and RBRs have interaction, specifically the two members of the Ariadne subfamily HHARI and TRIAD. Autoubiquitination assays show that adding NEDD8ylated Cul1Rbx 1 activated wild type HHARI significantly, which is normally autoinhibited (Kelsall et al., 2013). NEDD8ylation of cullin RING ligase is essential for the activity of CRLs, and Kelsall et al were able to discover that only the NEDD8ylated cullin RING ligases activate HHARI, and the unmodified form of CRLs have no effect. In fact, modification of CRL by the ubiquitin like protein NEDD8 causes dramatic reorientation of the structure allowing conformations capable of substrate polyubiquitination (Duda et al., 2008). The interaction between these two types E3 ligases opens up possibilities of a whole new mechanism of the function of E3s. In fact, another type of RBR Parc is known to be a fused form of Cul7 and an RBR Ariadne gene, which indicates that interaction of cullins and HHARI might have specific functions (Skaar et al., 2007). 


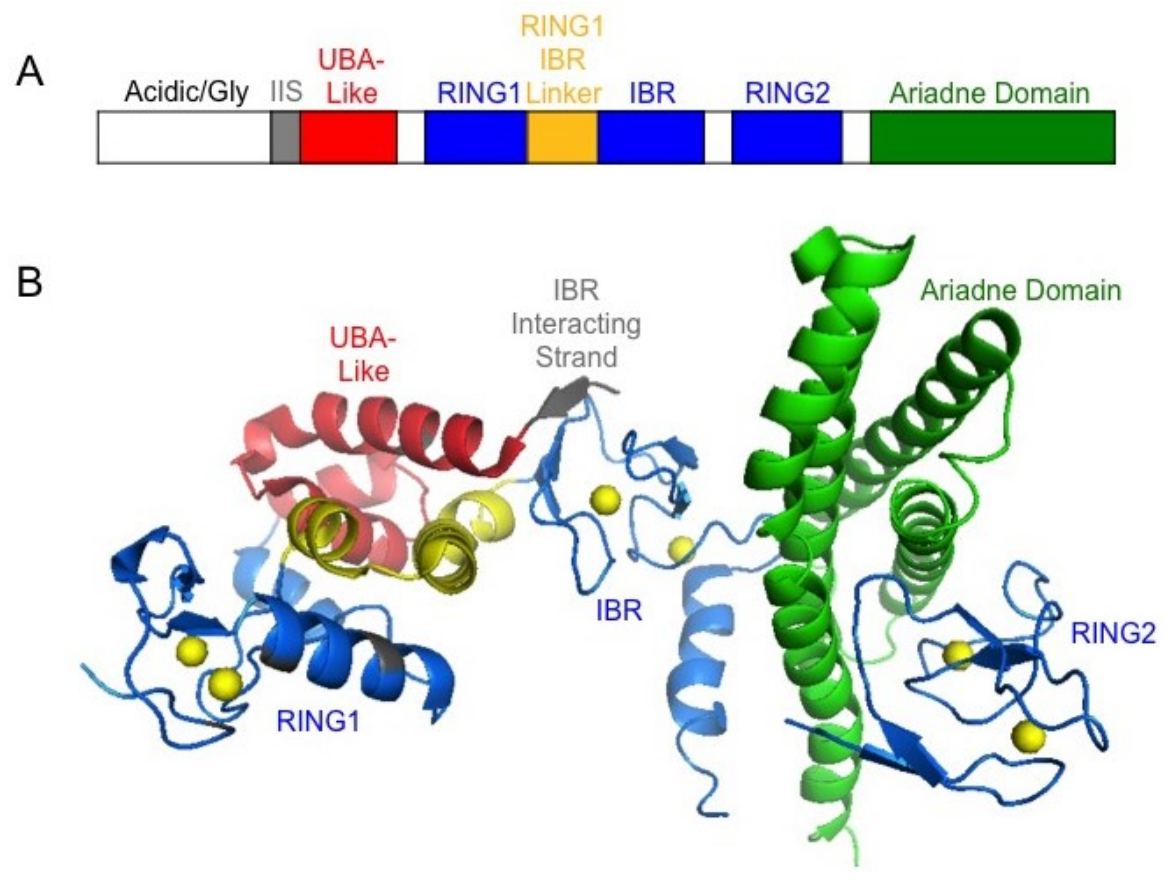

Figure 1-2. Human Homologue of Ariadne.

(A) Domains of HHARI, human homolog of Ariadne. HHARI has an Acidic/Gly rich domain, UBA domain, RING1, IBR, RING2, and an inhibitory Ariadne domain. (B) Structure of HHARI. The Ariadne domain covers about $30 \%$ of RING2, containing the catalytic site. Circles in yellow indicate zinc ions.

Data source: DOI: $10.2210 / \mathrm{pdb} 4 \mathrm{kbl} / \mathrm{pdb}$ Duda, D.M., Olszewski, J.L., Schuermann, J.P., Kurinov, I., Miller, D.J., Nourse, A., Alpi, A.F., and Schulman, B.A. (2013). Structure of HHARI, a RING-IBR-RING Ubiquitin Ligase: Autoinhibition of an Ariadne-Family E3 and Insights into Ligation Mechanism. Structure 21, 1030-1041. 


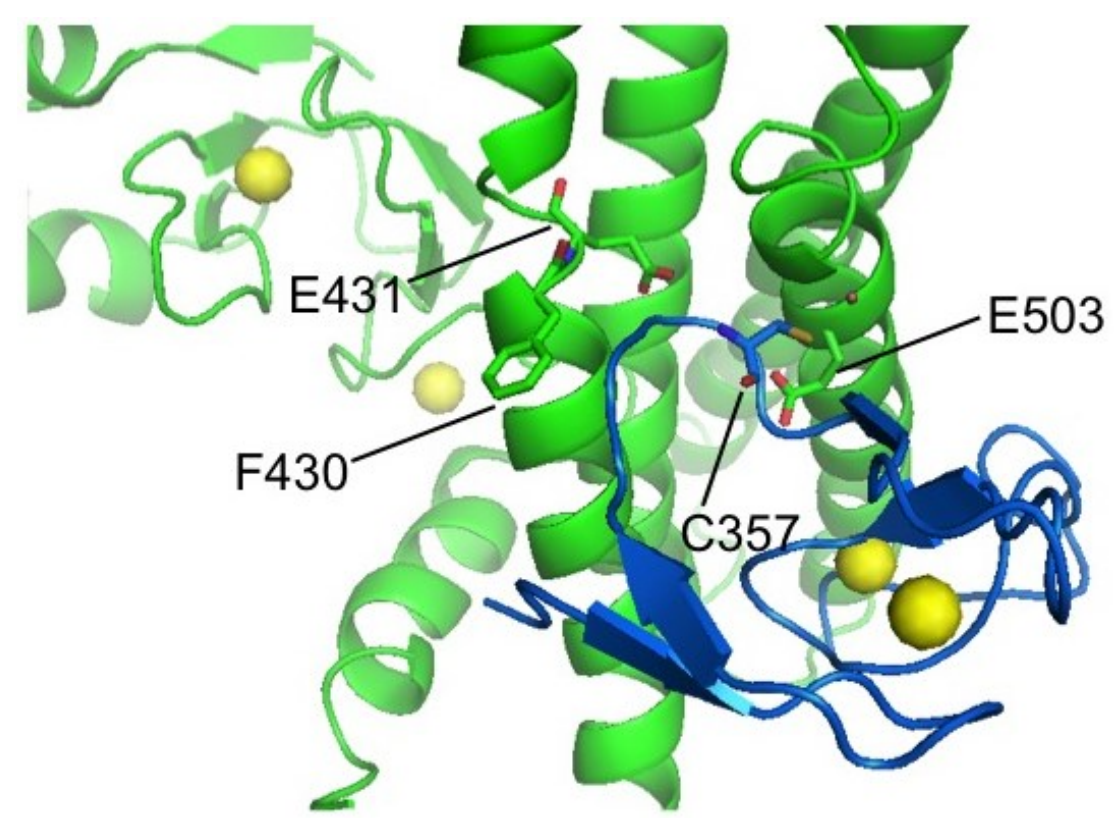

Figure 1-3. FEE Mutation of HHARI.

Sites of FEE mutation of HHARI. F430A, E431A, and E503A are sites closely related to the catalytic cysteine $\mathrm{C} 357$. Circles in yellow indicate zinc ions.

Data Source: DOI: $10.2210 / \mathrm{pdb} 4 \mathrm{kbl} / \mathrm{pdb}$

Duda, D.M., Olszewski, J.L., Schuermann, J.P., Kurinov, I., Miller, D.J., Nourse, A., Alpi, A.F., and Schulman, B.A. (2013). Structure of HHARI, a RING-IBR-RING Ubiquitin Ligase: Autoinhibition of an Ariadne-Family E3 and Insights into Ligation Mechanism. Structure 21, 1030-1041. 
Based on these evidences, my primary goal is to study this complex of two E3 ligases structurally to understand its mechanism. As technology for electron microscopy is on its prime time of development, I can take advantage of this to study the structural interaction between the two proteins. Now, electron microscopy can produce 3D images of macromolecules with resolutions close to that of X-ray crystallography or NMR spectroscopy. Another groundbreaking technology of EM microscopy called GraFix, which artificially fixes a complex during ultracentrifugation will facilitate greatly in trying to stabilize the complex during electron microscopy. Capturing the NEDD8ylated cullin and HHARI in action, along with the E2 and a potential substrate reveal the molecular basis for a fundamentally novel mechanism of E3 ligases. 


\section{CHAPTER 2. METHODS}

\section{Protein Expression and Purification}

All proteins used correspond to human sequences. Full length HHARI and F430A, E431A, E503A triple mutant were both cloned into pGEX4T1 (GE) modified with a TEV proteolytic cleavage site following GST. HHARI wt and FEE clones were transformed to BL21 (DE3) Gold competent cells for expression in E.coli. Cultures were grown in LB media in the presence of antibiotics at $37^{\circ} \mathrm{C}$ shaking at $200 \mathrm{rpm}$. At O.D. of 0.8 , cells were induced with $0.6 \mathrm{mM}$ IPTG (isopropyl beta-D-thiogalactopyranoside) and grown overnight at $16^{\circ} \mathrm{C}$. Proteins were purified by glutathione affinity chromatography, followed by overnight TEV proteolysis at $4^{\circ} \mathrm{C}$ to separate HHARI and GST. HHARI was purified away from GST by anion exchange with a $5 \mathrm{ml}$ High trap Q column (GE), and further purified by size exclusion chromatography in buffer conditions of 25mM HEPES $\mathrm{pH} 7.5,150 \mathrm{mM} \mathrm{NaCl}, 1 \mathrm{mM}$ DTT. After size-exclusion chromatography, proteins were pooled, concentrated, flash-frozen, and stored at $-80^{\circ} \mathrm{C}$. $\mathrm{UbcH} 7, \mathrm{Uba} 1$, and ubiquitin used in the autoubiquitination assays along with APPBP1-Uba3, Ubc12, and Nedd8 used in NEDD8ylation reactions were prepared in a similar manner.

Constructs of full-length Cull and Rbx1 were cloned into pFastBac vectors, with untagged Cull and TEV cleavable GST-TEV-Rbx1. Baculoviruses were made with bacmid DNA transformed into DH10alpha, and were amplified in Sf9 insect cells. Cul1 and Rbx1 was coexpressed in High Five insect cells, using viruses of Cul1 and GSTRbx1. Cul1Rbx1 was purified by glutathione affinity chromatography, followed by overnight TEV proteolysis at $4^{\circ} \mathrm{C}$. Further purification was done by cation exchange with $5 \mathrm{ml}$ High trap S column, and size-exclusion chromatography in buffer conditions of 25mM HEPES pH 7.5, 150mM NaCl, 1mM DTT. After size exclusion-chromatography, proteins were pooled, concentrated, and flash-frozen, and stored at $-80^{\circ} \mathrm{C}$.

\section{Biochemical Reactions and Assays}

NEDD8ylation reactions of Cul1Rbx 1 and Cul3Rbx1 were done by incubating 100nM APPBP1-Uba3, $1 \mu \mathrm{M}$ Ubc12, $12 \mu \mathrm{M}$ Cul1Rbx1, $25 \mu \mathrm{M}$ Nedd8, in $25 \mathrm{mM}$ HEPES $\mathrm{pH} 7.8,100 \mathrm{mM} \mathrm{NaCl}, 2.5 \mathrm{mM} \mathrm{MgCl} 2,1 \mathrm{mM}$ ATP, for $9 \mathrm{~min}$ at room temperature. The reaction was done in $2 \mathrm{ml}$ volume, quenched with $10 \mathrm{mM}$ DTT. Sample was spun down $13,000 \mathrm{rpm}$ for $10 \mathrm{~min}$ and further purified by size-exclusion chromatography in $25 \mathrm{mM}$ HEPES pH 7.5, 150mM NaCl, 1mM DTT.

Autoubiquitination reactions were performed by incubating $200 \mathrm{nM}$ Uba1, $1 \mu \mathrm{M}$ UbcH7, 20 $\mu$ M Ubiquitin, 400nM HHARI, $\pm 400 \mathrm{nM}$ N8Cul1Rbx1/N8Cul3Rbx1 in 50mM Tris- $\mathrm{HCl} \mathrm{pH} 7.6,50 \mathrm{mM} \mathrm{NaCl}, 10 \mathrm{mM} \mathrm{MgCl}_{2}, 5 \mathrm{mM}$ ATP at room temperature, and was quenched with $2 X$ SDS-PAGE sample buffer containing $20 \mathrm{mM}$ DTT each time point. For reactions containing N8Cul1Rbx1/N8Cul3Rbx1, cullins were preincubated with HHARI for 20 min on ice. Reactions were initiated by adding Ubal and were run on 4\%- 
$12 \%$ gradient gels (Invitrogen), stained overnight by SYPRO-Ruby stain, and visualized by a Typhoon gel-imager.

\section{Analytical Size-Exclusion Chromatography}

Analytical size-exclusion chromatography was used to examine association between HHARI and NEDD8ylated cullin RING ligases. Proteins were mixed at equimolar concentrations of $10 \mu \mathrm{M}$ in $200 \mu \mathrm{l}$ volumes on ice for $30 \mathrm{~min}$. Each mix was spun down at $13000 \mathrm{rpm}$ for 10 minutes and loaded onto a SD200 10/300 column (GE), in buffer conditions of $25 \mathrm{mM}$ HEPES pH 7.5, $100 \mathrm{mM} \mathrm{NaCl}, 1 \mathrm{mM}$ DTT. $500 \mu 1$ fractions were collected and run on $10 \%$ SDS gels, and visualized by Coomassie staining.

\section{GraFix and Negative Staining}

Protein samples were mixed at equimolar concentrations of $10 \mu \mathrm{M}$ in $100 \mu 1$. N8Cul1Rbx 1, Skp1Fbw7, HHARI FEE mutant were mixed on ice for $30 \mathrm{~min}$. The sample was spun down $35,000 \mathrm{rpm}$ for $15 \mathrm{hr}$ in a $5 \mathrm{ml} \mathrm{10 \% -30 \% (w/v)} \mathrm{sucrose} \mathrm{gradient}$ made by $25 \mathrm{mM}$ HEPES $\mathrm{pH} 7.5,200 \mathrm{mM} \mathrm{NaCl}, 1 \mathrm{mM}$ DTT. The sucrose gradient contained $0.05 \%$ gluteraldehyde for fixation. $205 \mu \mathrm{l}$ fractions were taken from the top and mixed with $5 \mu 1$ of $0.5 \mathrm{M}$ Sodium Aspartate $\mathrm{pH} 8.0$ for each fraction. Fractions were measured with 1x Biorad protein assay on a 96well plate, and desired fractions were collected and flash frozen before use. $30 \mu 1$ of each fraction were used for making negative stain grids and fixed with $2 \%$ Uranyl acetate on carbon film. 


\section{CHAPTER 3. RESULTS}

\section{NEDD8ylated Cullin RING Ligases Activate HHARI}

I sought to understand the mechanisms of how E3 ligases gain activity, specifically RBR type E3 ligases. Studies by Duda et al gave us insight into how the F430A, E431A, E503A triple mutation helps activate HHARI. Further studies from Kelsall et al showed that adding NEDD8ylated cullin RING ligase results in HHARI activity. It might be possible that the interaction between the two E3 ligases might produce an entire new form of E3 that has its own certain mechanism for substrate ubiquitination. Therefore, I first tested whether the FEE mutation enhances interaction with the NEDD8ylated cullins by looking at the autoubiquitination activity of HHARI. HHARI FEE clearly showed activity compared to wild type HHARI by formation of ubiquitin chains on HHARI (Figure 3-1A). Adding either NEDD8ylated Cul1Rbx1 or Cul3Rbx1 both slightly activated HHARI WT. As HHARI FEE activates so quickly, there is not a noticeable difference in autoubiquitination when NEDD8ylated cullins are added (Figure 3-1B, C). However, it was clear from the wild type activation that the NEDD8ylated cullins influence HHARI activation.

\section{NEDD8ylated Cullin RING Ligases Bind with HHARI}

In order to understand how the NEDD8ylated cullin RING ligases interact with HHARI, I performed analytical size exclusion chromatography to assess complex formation. If the interaction enhances HHARI activity, it is possible that the two proteins have structural interaction. First, the complex formed with wild type HHARI and N8Cul1Rbx 1 produced a higher molecular weight complex, but also produced a big shoulder peak that seemed to have a partial product. However, HHARI FEE and N8Cul1Rbx 1 made a larger complex, with a smaller shoulder peak (Figure 3-2A). Seen by coomassie staining of gels run on same fractions, both WT or FEE HHARI make a complex with N8Cul1Rbx1, but the FEE complex makes a larger form of almost two fractions (Figure 3-2B). This suggests that it is possible the HHARI FEE makes a tighter complex with N8Cul1Rbx1 compared to HHARI WT.

An analogous experiment showed that N8Cul3Rbx 1 also helps activate HHARI WT on its autoubiquitination. However, surprisingly there were no differences between the sizes of the complexes made between N8Cul3Rbx 1 and HHARI WT or HHARI FEE (Figure 3-3A, B). Therefore, the N8Cul3Rbx 1 did not differentiate whether HHARI has the FEE mutation or not. Overlap of all peaks showed that the largest complex made was between N8Cul1Rbx1 and HHARI FEE (Figure 3-3C). 

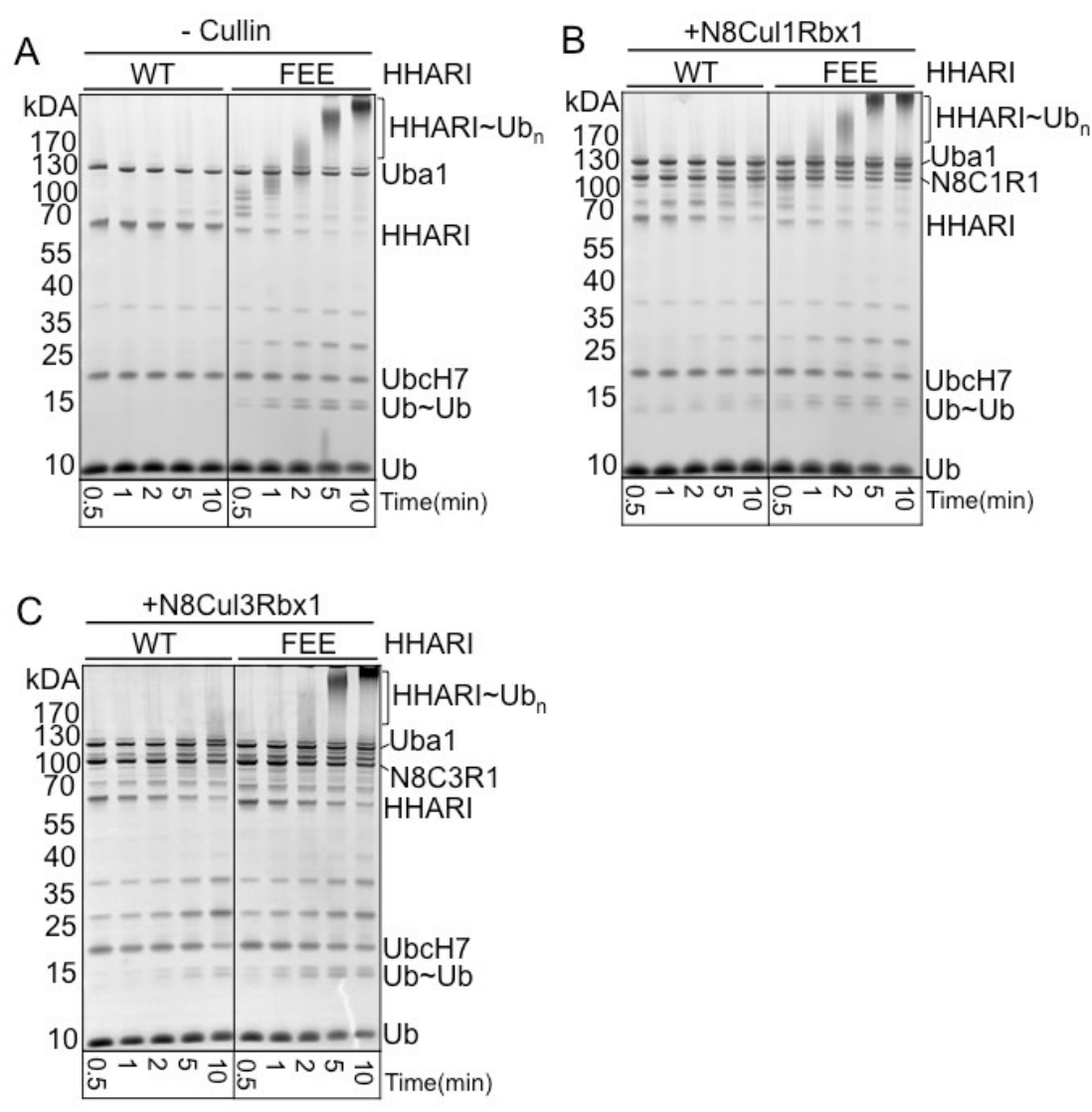

Figure 3-1. FEE Mutation of HHARI and the Addition of N8Cul1Rbx1 Both Activate HHARI.

(A) WT HHARI stays inactivate as no autoubiquitin chains are built by time. However, FEE mutation of HHARI induces autoubiquitination activity starting from $0.5 \mathrm{~min}$ building autoubiquitin chains, and almost fully saturated by $5 \mathrm{~min}$. (B) N8Cul1 Rbx 1 promotes wild type HHARI activation, while enhancing the activity of HHARI FEE mutant. (C) N8Cul3Rbx1 also promotes wild type HHARI activation, while enhancing the activity of HHARI FEE mutant. All assays are observed by SYPRO-Ruby staining. 

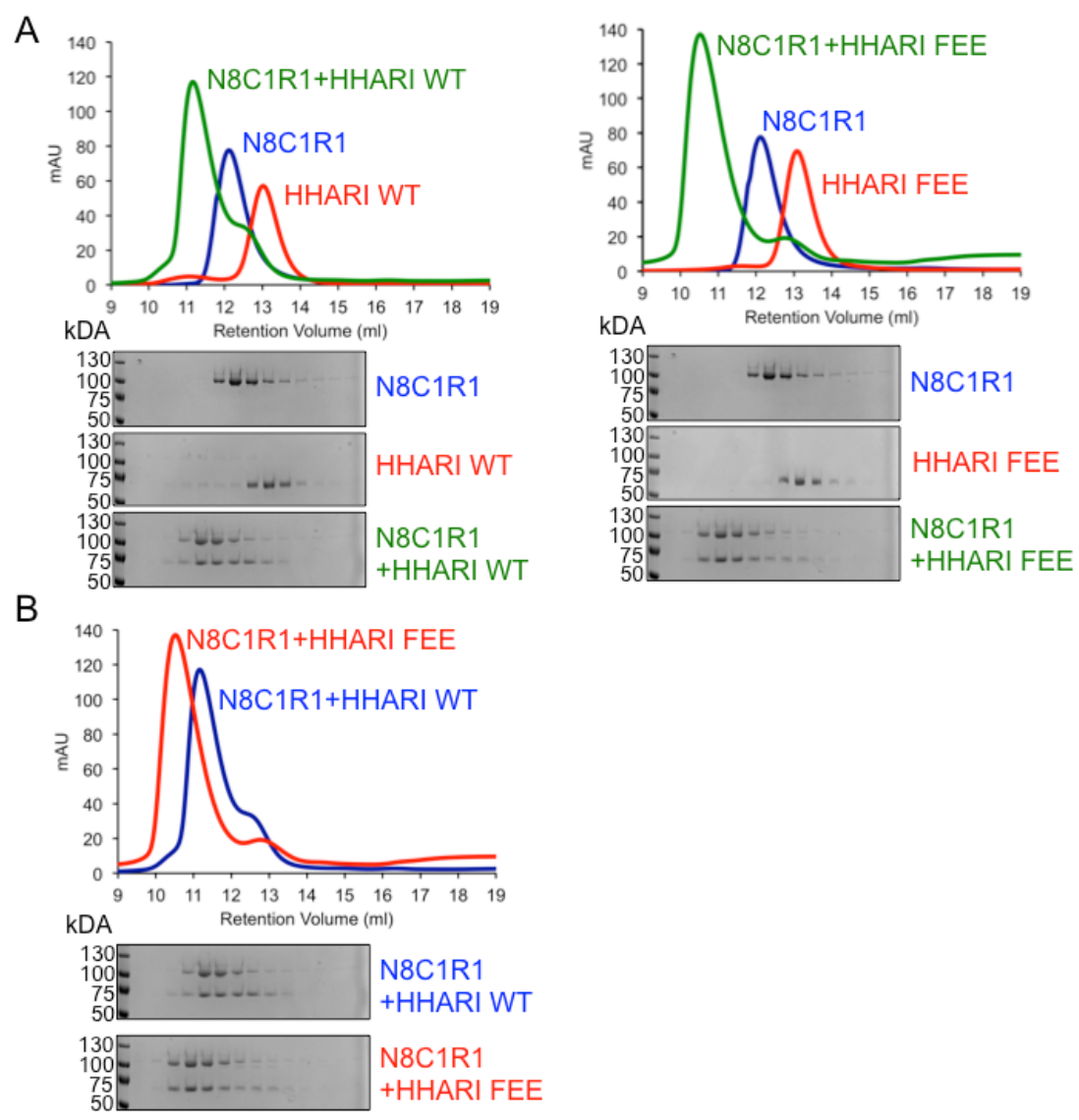

\section{Figure 3-2. Analytical Size-Exclusion Chromatography Shows Interaction Between N8Cul1Rbx1 and HHARI.}

(A) Wild type HHARI forms a complex with N8Cul1Rbx1, but makes a big shoulder peak of an intermediate complex. HHARI FEE mutant forms a larger complex with almost very little intermediate product, indicating equilibrium shifting more towards complex formation. (B) Overlay of two complexes made either by wild type or FEE mutant HHARI. FEE mutant clearly makes a better complex with N8Cul1Rbx1. 

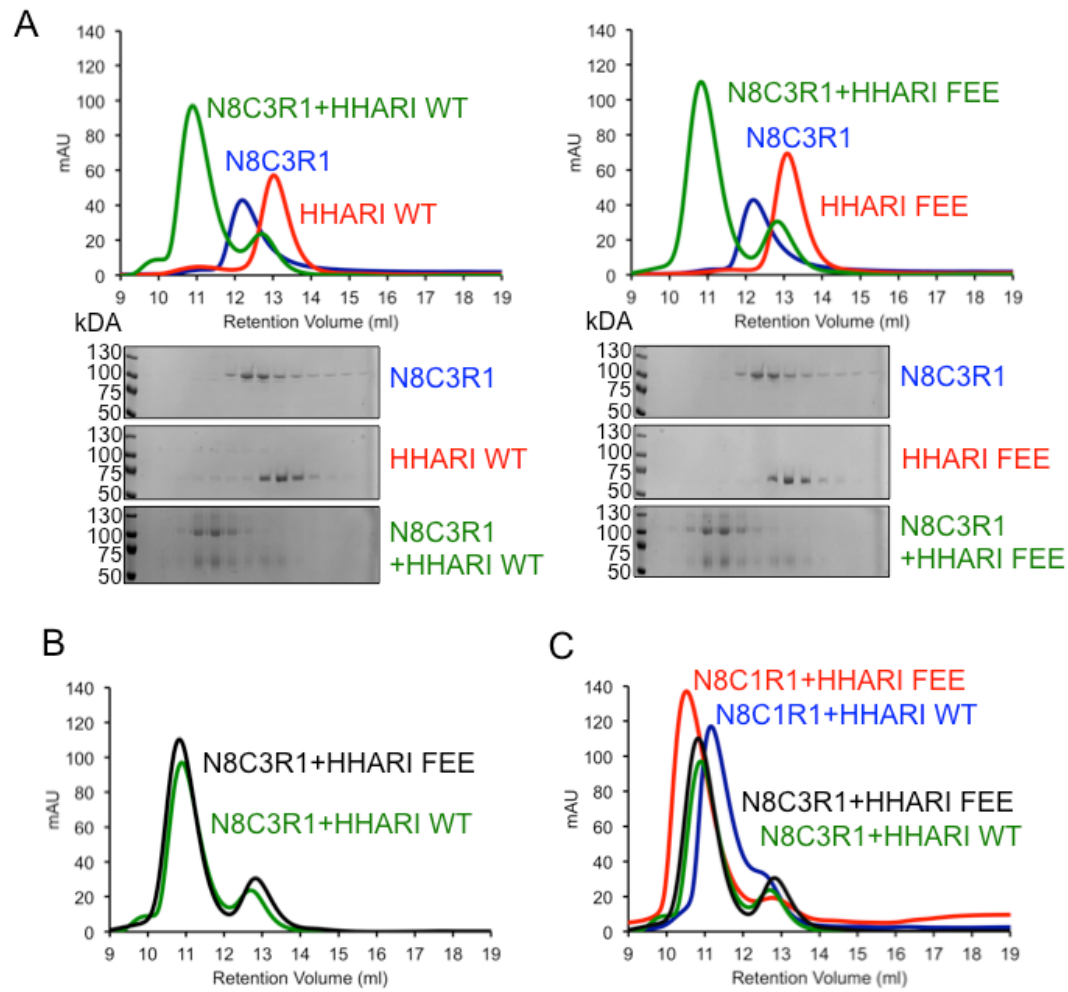

Figure 3-3. Interaction Between N8Cul3Rbx1 and HHARI.

(A) Both wild type and FEE mutant HHARI forms a complex with N8Cul3Rbx1 in a similar manner, making similar shoulder intermediates. (B) Overlay of two complexes made either by wild type or FEE mutant HHARI. They are identical. (C) Overall overlay all type of complexes. N8Cul1Rbx 1 and HHARI FEE form the largest complex with the least intermediate. 


\section{GraFix and Negative Staining}

We sought to study the newly formed complex by structural studies. However, due to the current limits of electron microscopy, a protein complex of less than $200 \mathrm{kDa}$ would not produce an ideal resolution. I tried to enhance contrast by making the complex bigger by adding an adaptor protein of N8Cul1Rbx1. Skp1Fbw7 is a substrate adaptor protein of N8Cul1Rbx 1 that dimerizes, suggesting the entire complex might be able to dimerize by Skp1Fbw7. However, adding Skp1Fbw7 to HHARI FEE and N8Cul1Rbx1 did not form a large complex when analyzed over size-exclusion chromatography (figure not shown). Therefore, another way to generate a robust complex was to artificially trap it. GraFix, a well known technique used to chemically lock a protein complex, was used for making samples for electron microscopy (Kastner et al., 2008). This allowed me to make a complex that contain all three proteins, HHARI FEE, N8Cul1Rbx 1, and Skp1Fbw7, possibly in a dimer form. The percentage of gluteraldehyde added, speed and time of centrifugation were optimized, calculated by the theoretical molecular weight.

The peaks of Biorad protein assay traveled to an optimal fraction after ultracentrifugation (Figure 3-4A).

Negative staining of samples of Fraction\#18 produced semi-homogenous molecular images (Figure 3-4B). We can definitely see the multiple circular shapes with a hollow center, and cryo EM is currently in progress. If the results produce a high resolution 3D image, it will give tremendous information on structural interaction between NEDD8ylated cullins and RBRs. 
A

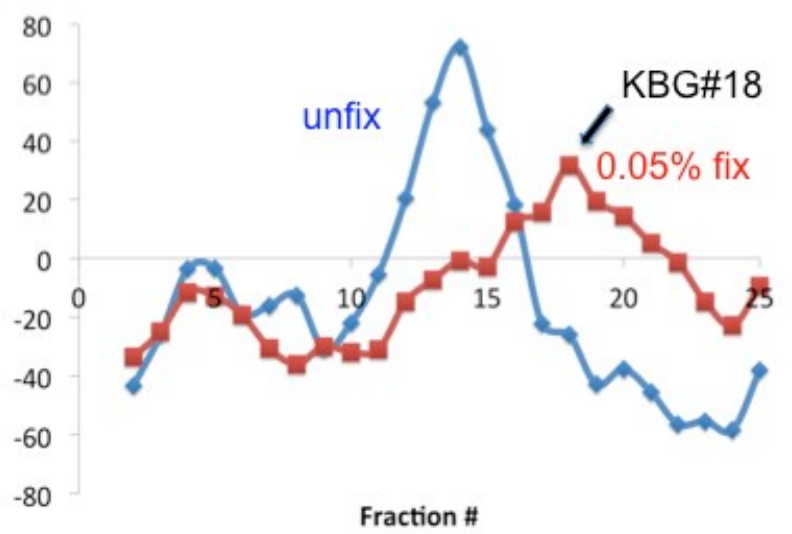

B

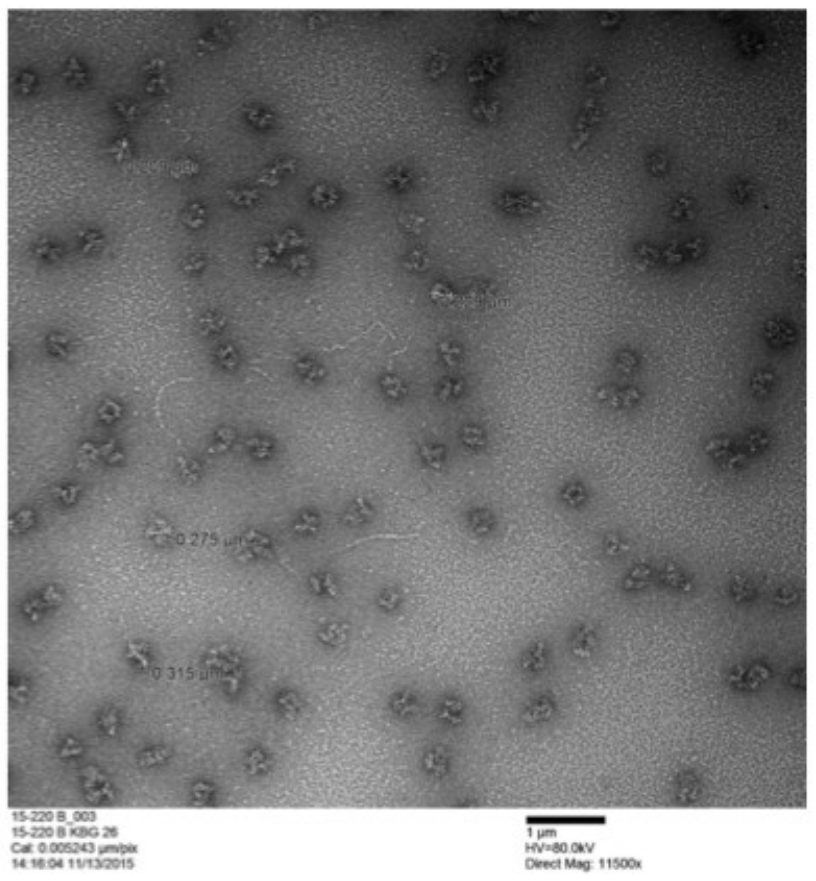

Figure 3-4. Electron Microscopy of HHARI FEE, N8Cul1Rbx1, and Skp1/Fbw7.

(A) $10-30 \%(\mathrm{w} / \mathrm{v})$ sucrose gradient ultracentrifugation fraction profile with or without 0.05\% Gluteraldehyde. Fraction\#18 was used for negative staining. (B) Negative stain image of Fraction\#18 of complex with HHARI FEE, N8Cul1Rbx1, and Skp1/Fbw7. 


\section{CHAPTER 4. DISCUSSION}

Here, I studied the interaction between two E3 ligases, HHARI and N8Cul1Rbx1. I showed that NEDD8ylated cullin RING ligases are able to make a complex with HHARI. A specific set of HHARI mutations, F430A, E431A, and E503A, was not only able to activate its autoubiquitination, but also produce a complex with N8Cul1Rbx1. Understanding the mechanism of the two E3 ligases' interaction may reveal a novel mechanism of the ubiquitin pathway and providing structural insights will be the key.

\section{Microscopic Reversibility of Protein Complex}

Microscopic reversibility states that there is always a reverse reaction by time, therefore a heterogenous substance can constantly exist in subgroups composed of intermediate states for every process (Lewis, 1925). So far, the biggest challenge in our efforts to make a stable complex with HHARI and N8CullRbx1 was that whenever the complex was run on size exclusion chromatography, it would produce a $70 \%$ bound form, with a shoulder peak that would indicate an unbound form or an intermediate. This resulted in many attempted failures on trying to crystallize the complex, and the crystals obtained were only one part of the complex, either HHARI or NEDD8ylated CullRbx1. Therefore the microscopic reversibility of the complex of N8Cul1Rbx 1 and HHARI WT has been an issue as the constant association and dissociation produced an unstable complex suitable for crystal formation. If we are able to somehow shift the equilibrium to where it is more favorable in a bound form, that would enable us to study its structure either through crystallography or electron microscopy.

\section{Stabilizing the Complex}

From preliminary studies (not shown), we know that the N-terminal 90 residues of HHARI, which are highly acidic and glycine rich, are necessary for binding to N8CullRbx 1. I believe the acidic regions are capable of making interactions but the abundance of glycines makes the structure not stable enough for a single, specific interaction. Therefore, this might be the reasoning behind the dynamic equilibrium state, producing intermediate products. It was surprising to find out that introducing the F430A, E431A, E503A triple mutation of HHARI was able to make a larger complex from size exclusion chromatography, while having a very little shoulder peak. This indicated that the equilibrium greatly shifted to the complex formation side and producing less intermediate forms than previous experiments. It is possible that the triple mutation opens up a secondary docking site for the NEDD8ylated cullin to bind. With the equilibrium shift, I was able to gain confidence that HHARI FEE form a much more stable complex compared to HHARI WT. 


\section{Ub-VME as a Chemical Warhead}

Chemically modified ubiquitins were initially introduced as probes for reactivity of deubiquitinating enzymes (DUB) (Borodovsky et al., 2002). In previous studies of Parkin, chemically modified ubiquitin-vinyl sulfone (Ub-VS) was implemented as a probe that covalently modifies the catalytic cysteine of Parkin without the help of E2 (Riley et al., 2013). Kelsall et al also implemented an electrophilic Ubiquitin-vinyl methyl ester (Ub-VME) to find that the Ub-VME binds to the catalytic cysteine of HHARI by either removing the Ariadne domain or the addition of NEDD8ylated cullins (Kelsall et al., 2013). In fact, there have been structural studies using the Ub-VME to study molecular contacts for ubiquitin recognition (Sheedlo et al., 2015). Implementing Ub-VME on structural studies of the complex with NEDD8ylated cullins and HHARI will have a modified ubiquitin bound to the active site cysteine, producing an active form of the complex. Further on, if this active site bound Ub-VME interacts with multiple sites on either HHARI or NEDD8ylated cullin, it can act as a chemical linker that locks the complex in action.

\section{Targets for Electron Microscopy}

Currently, size is a very important factor in achieving high-resolution 3D images for electron microscopy. Therefore, dimerizing the complex would significantly improve EM studies. F-box protein Fbw7 of Cul1Rbx1 would theoretically produce a dimer. Also, Cul3Rbx1 binds with a BTB protein that is known to interact with substrate adaptors. Most importantly they are known to dimerize (Furukawa et al., 2003; Zhuang et al., 2009). As Cul3Rbx1 also interacts with HHARI, dimerization of Cul3Rbx1 and its substrate adaptor will likely cause dimerization of the potentially HHARI bound complex as well, producing a sizable protein suitable for electron microscopy. However, according to the analytical size-exclusion chromatography experiments (Figure 6B), N8Cul3Rbx1 did not make a difference in the interaction between either HHARI WT or FEE mutant. Therefore, it is not clear whether N8Cul3Rbx1 would interact in a similar way with N8Cul1Rbx 1, but it will definitely give insight towards how the cullin RING ligases interact with HHARI.

According to protein interaction studies, HHARI is seen to interact with Cul1, $\mathrm{Cul2,} \mathrm{Cul3}$, and Cul4A, which can also be tried for structural studies. Another Ariadne family protein TRIAD1 can also be used, as Kelsall et al used to study interactions with Cul5. One option to study a complex between RBR and cullins would be Parc (Cu19) as this is known as a cullin and Ariadne RBR fused protein. So far, there have been no structural studies on this protein, which can potentially provide great insight on the cullin RBR complex. From these studies I hope to learn a novel mechanism of two different E3 ligases working together. It is possible that the study might reveal a new category of E3 ligases, as there have been no studies on this interaction mechanism. Further on, as an E3 ligase, its substrate regulation will be most important in its participation in cellular processes. It will be interesting to study what this new complex regulates downstream, 
whether it will affect substrates of NEDD8ylated cullins, HHARI, or affect entirely new substrates. 


\section{LIST OF REFERENCES}

Borden, K.L., Boddy, M.N., Lally, J., O'Reilly, N.J., Martin, S., Howe, K., Solomon, E., and Freemont, P.S. (1995). The solution structure of the RING finger domain from the acute promyelocytic leukaemia proto-oncoprotein PML. The EMBO journal 14, 15321541.

Borodovsky, A., Ovaa, H., Kolli, N., Gan-Erdene, T., Wilkinson, K.D., Ploegh, H.L., and Kessler, B.M. (2002). Chemistry-based functional proteomics reveals novel members of the deubiquitinating enzyme family. Chemistry \& biology 9, 1149-1159.

Cardozo, T., and Pagano, M. (2004). The SCF ubiquitin ligase: insights into a molecular machine. Nat Rev Mol Cell Biol 5, 739-751.

Cavadini, S., Fischer, E.S., Bunker, R.D., Potenza, A., Lingaraju, G.M., Goldie, K.N., Mohamed, W.I., Faty, M., Petzold, G., Beckwith, R.E., et al. (2016). Cullin-RING ubiquitin E3 ligase regulation by the COP9 signalosome. Nature 531, 598-603.

Chaugule, V.K., Burchell, L., Barber, K.R., Sidhu, A., Leslie, S.J., Shaw, G.S., and Walden, H. (2011). Autoregulation of Parkin activity through its ubiquitin-like domain. The EMBO journal 30, 2853-2867.

Deshaies, R.J., and Joazeiro, C.A. (2009). RING domain E3 ubiquitin ligases. Annu Rev Biochem 78, 399-434.

Duda, D.M., Borg, L.A., Scott, D.C., Hunt, H.W., Hammel, M., and Schulman, B.A. (2008). Structural insights into NEDD8 activation of cullin-RING ligases: conformational control of conjugation. Cell 134, 995-1006.

Duda, D.M., Olszewski, J.L., Schuermann, J.P., Kurinov, I., Miller, D.J., Nourse, A., Alpi, A.F., and Schulman, B.A. (2013). Structure of HHARI, a RING-IBR-RING Ubiquitin Ligase: Autoinhibition of an Ariadne-Family E3 and Insights into Ligation Mechanism. Structure 21, 1030-1041.

Eisenhaber, B., Chumak, N., Eisenhaber, F., and Hauser, M.T. (2007). The ring between ring fingers (RBR) protein family. Genome Biol 8, 209.

Enchev, R.I., Scott, D.C., da Fonseca, P.C., Schreiber, A., Monda, J.K., Schulman, B.A., Peter, M., and Morris, E.P. (2012). Structural basis for a reciprocal regulation between SCF and CSN. Cell Rep 2, 616-627.

Furukawa, M., He, Y.J., Borchers, C., and Xiong, Y. (2003). Targeting of protein ubiquitination by BTB-Cullin 3-Roc1 ubiquitin ligases. Nat Cell Biol 5, 1001-1007. 
Hodgins, R.R., Ellison, K.S., and Ellison, M.J. (1992). Expression of a ubiquitin derivative that conjugates to protein irreversibly produces phenotypes consistent with a ubiquitin deficiency. The Journal of biological chemistry 267, 8807-8812.

Hristova, V.A., Beasley, S.A., Rylett, R.J., and Shaw, G.S. (2009). Identification of a novel $\mathrm{Zn} 2+-$ binding domain in the autosomal recessive juvenile Parkinson-related E3 ligase parkin. The Journal of biological chemistry 284, 14978-14986.

Huang, D.T., Ayrault, O., Hunt, H.W., Taherbhoy, A.M., Duda, D.M., Scott, D.C., Borg, L.A., Neale, G., Murray, P.J., Roussel, M.F., et al. (2009). E2-RING expansion of the NEDD8 cascade confers specificity to cullin modification. Molecular cell 33, 483-495.

Huibregtse, J.M., Scheffner, M., Beaudenon, S., and Howley, P.M. (1995). A family of proteins structurally and functionally related to the E6-AP ubiquitin-protein ligase.

Proceedings of the National Academy of Sciences of the United States of America 92, 2563-2567.

Kastner, B., Fischer, N., Golas, M.M., Sander, B., Dube, P., Boehringer, D., Hartmuth, K., Deckert, J., Hauer, F., Wolf, E., et al. (2008). GraFix: sample preparation for singleparticle electron cryomicroscopy. Nat Methods 5, 53-55.

Kelsall, I.R., Duda, D.M., Olszewski, J.L., Hofmann, K., Knebel, A., Langevin, F., Wood, N., Wightman, M., Schulman, B.A., and Alpi, A.F. (2013). TRIAD1 and HHARI bind to and are activated by distinct neddylated Cullin RING ligase complexes. The EMBO journal.

Komander, D. (2009). The emerging complexity of protein ubiquitination. Biochem Soc Trans 37, 937-953.

Komander, D., and Rape, M. (2012). The ubiquitin code. Annu Rev Biochem 81, 203229.

Koyano, F., Okatsu, K., Kosako, H., Tamura, Y., Go, E., Kimura, M., Kimura, Y., Tsuchiya, H., Yoshihara, H., Hirokawa, T., et al. (2014). Ubiquitin is phosphorylated by PINK1 to activate parkin. Nature 510, 162-166.

Lewis, G.N. (1925). A New Principle of Equilibrium. Proceedings of the National Academy of Sciences of the United States of America 11, 179-183.

Lyapina, S., Cope, G., Shevchenko, A., Serino, G., Tsuge, T., Zhou, C., Wolf, D.A., Wei, N., Shevchenko, A., and Deshaies, R.J. (2001). Promotion of NEDD-CUL1 conjugate cleavage by COP9 signalosome. Science 292, 1382-1385.

Lydeard, J.R., Schulman, B.A., and Harper, J.W. (2013). Building and remodelling Cullin-RING E3 ubiquitin ligases. EMBO Rep 14, 1050-1061. 
Mosadeghi, R., Reichermeier, K.M., Winkler, M., Schreiber, A., Reitsma, J.M., Zhang, Y., Stengel, F., Cao, J., Kim, M., Sweredoski, M.J., et al. (2016). Structural and kinetic analysis of the COP9-Signalosome activation and the cullin-RING ubiquitin ligase deneddylation cycle. eLife 5 .

Pierce, N.W., Lee, J.E., Liu, X., Sweredoski, M.J., Graham, R.L., Larimore, E.A., Rome, M., Zheng, N., Clurman, B.E., Hess, S., et al. (2013). Cand1 promotes assembly of new $\mathrm{SCF}$ complexes through dynamic exchange of $\mathrm{F}$ box proteins. Cell 153, 206-215.

Riley, B.E., Lougheed, J.C., Callaway, K., Velasquez, M., Brecht, E., Nguyen, L., Shaler, T., Walker, D., Yang, Y., Regnstrom, K., et al. (2013). Structure and function of Parkin E3 ubiquitin ligase reveals aspects of RING and HECT ligases. Nat Commun 4, 1982.

Saha, A., and Deshaies, R.J. (2008). Multimodal activation of the ubiquitin ligase SCF by Nedd8 conjugation. Molecular cell 32, 21-31.

Schmidt, M.W., McQuary, P.R., Wee, S., Hofmann, K., and Wolf, D.A. (2009). F-boxdirected CRL complex assembly and regulation by the CSN and CAND1. Molecular cell 35, 586-597.

Schulman, B.A., Carrano, A.C., Jeffrey, P.D., Bowen, Z., Kinnucan, E.R., Finnin, M.S., Elledge, S.J., Harper, J.W., Pagano, M., and Pavletich, N.P. (2000). Insights into SCF ubiquitin ligases from the structure of the Skp1-Skp2 complex. Nature 408, 381-386.

Schulman, B.A., and Harper, J.W. (2009). Ubiquitin-like protein activation by E1 enzymes: the apex for downstream signalling pathways. Nat Rev Mol Cell Biol 10, 319331.

Scott, D.C., Sviderskiy, V.O., Monda, J.K., Lydeard, J.R., Cho, S.E., Harper, J.W., and Schulman, B.A. (2014). Structure of a RING E3 Trapped in Action Reveals Ligation Mechanism for the Ubiquitin-like Protein NEDD8. Cell 157, 1671-1684.

Sheedlo, M.J., Qiu, J., Tan, Y., Paul, L.N., Luo, Z.Q., and Das, C. (2015). Structural basis of substrate recognition by a bacterial deubiquitinase important for dynamics of phagosome ubiquitination. Proceedings of the National Academy of Sciences of the United States of America 112, 15090-15095.

Skaar, J.R., Florens, L., Tsutsumi, T., Arai, T., Tron, A., Swanson, S.K., Washburn, M.P., and DeCaprio, J.A. (2007). PARC and CUL7 form atypical cullin RING ligase complexes. Cancer research 67, 2006-2014.

Spratt, D.E., Walden, H., and Shaw, G.S. (2014). RBR E3 ubiquitin ligases: new structures, new insights, new questions. Biochem J 458, 421-437.

Streich, F.C., Jr., and Lima, C.D. (2014). Structural and functional insights to ubiquitinlike protein conjugation. Annual review of biophysics 43, 357-379. 
Tang, X., Orlicky, S., Lin, Z., Willems, A., Neculai, D., Ceccarelli, D., Mercurio, F., Shilton, B.H., Sicheri, F., and Tyers, M. (2007). Suprafacial orientation of the SCFCdc4 dimer accommodates multiple geometries for substrate ubiquitination. Cell 129, 1165 1176.

Trempe, J.F., Sauve, V., Grenier, K., Seirafi, M., Tang, M.Y., Menade, M., Al-AbdulWahid, S., Krett, J., Wong, K., Kozlov, G., et al. (2013). Structure of Parkin Reveals Mechanisms for Ubiquitin Ligase Activation. Science 340, 1451-1455.

Wauer, T., and Komander, D. (2013). Structure of the human Parkin ligase domain in an autoinhibited state. The EMBO journal.

Wenzel, D.M., Lissounov, A., Brzovic, P.S., and Klevit, R.E. (2011). UBCH7 reactivity profile reveals parkin and HHARI to be RING/HECT hybrids. Nature 474, 105-108.

Zhuang, M., Calabrese, M.F., Liu, J., Waddell, M.B., Nourse, A., Hammel, M., Miller, D.J., Walden, H., Duda, D.M., Seyedin, S.N., et al. (2009). Structures of SPOP-substrate complexes: insights into molecular architectures of BTB-Cul3 ubiquitin ligases.

Molecular cell 36, 39-50.

Zimmerman, E.S., Schulman, B.A., and Zheng, N. (2010). Structural assembly of cullinRING ubiquitin ligase complexes. Curr Opin Struct Biol 20, 714-721. 


\section{VITA}

Kheewoong Baek was born in 1990, Daegu, Korea. After receiving his high school diploma from Hankuk Academy of Foreign Studies, Korea, he entered Rutgers University in New Jersey. He graduated December of 2012 with a Bachelor of Arts in Biological Sciences. Following February, he started a postbaccalaureate program at NIH in the lab of Dr. Yihong Ye. 2014, he joined the school of Graduate Health Sciences at University of Tennessee Health Science Center, carrying on his Masters studies in the lab of Dr. Brenda A. Schulman at St.Jude Children's Research Hospital. In May of 2016, he will receive his Master of Science degree from the University of Tennessee. 